\title{
WEAK FORMULATION STUDY FOR THERMOELASTIC BUCKLING ANALYSIS OF THICK LAMINATED CYLINDRICAL SHELLS
}

\author{
DING Kewei \\ School of Civil Engineering, Anhui Jianzhu University \\ Hefei 230601, Anhui, P.R. CHINA \\ E-mail:dingkw@ahjzu.edu.cn
}

\begin{abstract}
Weak formulations of mixed state equations of closed laminated cylindrical shells are presented in the Hamilton System. The Hamilton canonical equation of closed cylindrical shell is established. By means of applying the transfer matrix method and taking the advantage of Hamiltonian matrix in the calculation, a unified approach and three-dimensional thermoelastic solutions are obtained for the buckling analysis of closed thick laminated cylindrical shells. All equations of elasticity can be satisfied and all elastic constants can be taken into account. Numerical results are given to compare with those of FEM calculated using SAP5.The principle and method suggested here have clear physical concepts. The equations and boundary conditions proposed in this paper are weakened. The solutions and results given here may serve as a benchmark for other numerical procedures.
\end{abstract}

Key words: weak formulations, buckling analysis, laminated cylindrical shells, thermoelastic, Hamilton canonical equation.

\section{Introduction}

The rapidly growing applications of fibre-reinforced composites in high-performance aerospace vehicles have led to an intensive study of the behavior of laminated composite structures under various conditions. The exact analysis of cylindrical shells have received widespread attention in recent years (Reddy, 1983; 1984; Srinivas and Rao, 1970). With no initial assumptions regarding stress and deformation models (Kardomateas, 1993; Soldatos and Hadjigeorgiou, 1990; Kawkes and Soldatos, 1992; Noor and Burton, 1996), and using three-dimensional elasticity the free vibration problems of homogeneous isotropic, orthotropic, and laminated thick cylindrical shells and plate were solved. Recently, three-dimensional static, dynamic, thermoelastic and buckling analysis of homogeneous and laminated composite cylinders have been studied by Soldatos and Ye (1994). Huang and Tauchert (1992) examined the thermal stresses in doublecurved cross-ply laminates. Chen and Liu (1993) discussed an analytical levy-type solution for thermal buckling of antisymmetric angle-ply laminated plates. Khdeir (1996) investigated thermal deformations and stresses in cross-ply laminated circular cylindrical shells by means of state space approach. Ding and Tang (1997; 1999; 1998) developed the method of state space, and gave the exact solution for axisymmetric vibration and buckling of laminated cylindrical shells having simply supported edge boundary and clamped edges, respectively. An exact thermoelastic solution for an axisymmetric problem of thick closed laminated shells has also been studied by Ding and Tang (1999). Ding $(2005 ; 2008 ; 2008)$ obtained three-dimensional solutions of thick closed laminated shell by means of weak formulation study. Hedayati et al. (2014), investigated semi-analytical 3D elasticity solutions for open cylindrical shells under various boundary conditions. The stability analysis and active control of a composite laminated open cylindrical shell in subsonic airflow is made by Yao Guo and Li Feng-Ming (2014). Topal Umut and Uzman Umit deals with frequency optimization of symmetrically laminated skewed open cylindrical shells (2011). A free vibration analysis of laminated conical shells is presented by Civalek Omer (2013) using the numerical solution of 
governing differential equations of motion based on transverse shear deformation theory. Approaches to modifying and controlling the elastic response of axially compressed laminated composite cylindrical shells in the far postbuckling regime are presented and evaluated by Burgueño Rigoberto, Hu Nan, Heeringa Annelise and Lajnef Nizar (2014). Free dynamic responses of a symmetrically laminated composite shell are used to analyze a nonlinear differential panel by Hashemian and Jam (2010). A thermal bending analysis of doubly curved laminated shell panels with general boundary conditions and laminations is presented by Maghami and Tahani (2015). Khdeir (2012) investigated thermal deformations in cross-ply laminated shells by state space approach. The effect of the through-the-thickness temperature distribution on the response of layered and composite shells is calculated by Brischetto (2009).

To the author's knowledge, three-dimensional buckling analysis for the quite thick closed laminated cylindrical shells is so difficult that few references have been found. In this paper, however, based upon weak formulation of mixed state equations including boundary conditions, the Hamilton canonical equation of closed laminated cylindrical shell is established. A three-dimensional solution is expressed for the buckling analysis of the thick closed laminated shell of arbitrary thickness. Numerical results are obtained and compared with those of FEM calculated using SAP5.

\section{Weak formulation}

An orthotropic closed cylindrical shell is shown in Fig.1. The principal elastic directions of the shell coincide with the coordinate axes. Let $u, v$ and $w$ be the displacement in the $x-, \theta$-and r-directions, respectively.
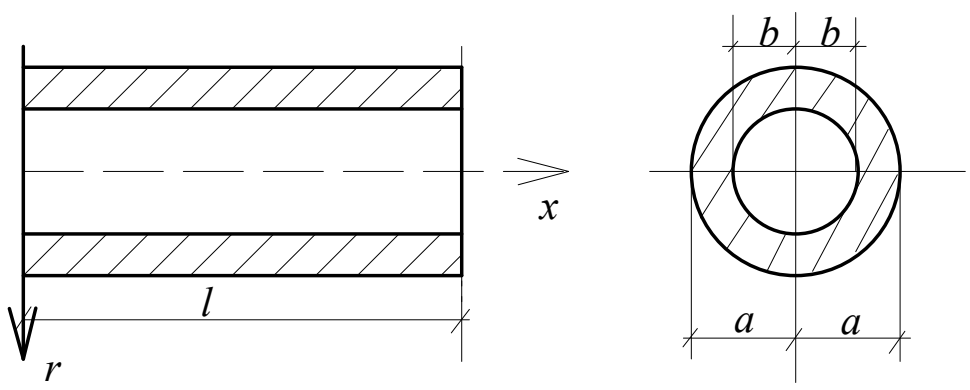

Fig.1. A closed cylindrical shell.

The modified mixed variational principle can be shown to be of the form

$$
\begin{aligned}
& \boldsymbol{\Pi}=\iiint_{v}\left\{\sigma_{r} \frac{\partial w}{\partial r}+\tau_{r \theta} \frac{\partial v}{\partial r}+\tau_{r x} \frac{\partial u}{\partial r}-H\right\} r d r d \theta d x+ \\
& -\iint_{s_{u}}\left[p_{r}(w-\bar{w})+p_{\theta}(v-\bar{v})+p_{x}(u-\bar{u})\right] d s-\iint_{s_{\sigma}}\left(\bar{p}_{r} w+\bar{p}_{\theta} v+\bar{p}_{x} u\right) d s
\end{aligned}
$$

in which the usual index notation is used. $S_{\sigma}$ and $S_{u}$ denote respectively the portion of the edge boundary where tractions $\bar{p}_{i}$ are prescribed and where displacements $\bar{u}_{i}$ are prescribed. The quadratic form of the Hamilton function $\mathrm{H}$ can be written as 


$$
\begin{aligned}
& -H=\sigma_{x}\left(\frac{\partial u}{\partial x}-\alpha_{x} T\right)+\sigma_{\theta}\left(\frac{w}{r}+\frac{1}{r} \frac{\partial v}{\partial \theta}-\alpha_{x} T\right)+\tau_{x \theta}\left(\frac{\partial v}{\partial x}+\frac{1}{r} \frac{\partial u}{\partial \theta}\right)+\sigma_{r}\left(-\alpha_{r} T\right)+ \\
& +\tau_{r \theta}\left(\frac{1}{r} \frac{\partial w}{\partial \theta}-\frac{v}{r}\right)+\tau_{r x} \frac{\partial w}{\partial x}-\frac{1}{2}\{\sigma\}^{T}[C]^{-1}\{\sigma\}-\frac{1}{2} p_{x}\left(\left(\frac{\partial u}{\partial x}\right)^{2}+\left(\frac{\partial v}{\partial x}\right)^{2}+\left(\frac{\partial w}{\partial x}\right)^{2}\right)
\end{aligned}
$$

where $\alpha_{x}, \alpha_{\theta}$ and $\alpha_{r}$ are the coefficients of thermal expansion, $T$ is the temperature rise from the stress-free state, $p_{x}$ are the uniformly distributed compressive stresses acting on the two edges of a shell, the matrix $[C]$ is the elastic stiffness matrix.

Letting $\delta \Pi=0$, yields

$$
\begin{aligned}
& \frac{\partial u}{\partial r}=\frac{\partial \mathrm{H}}{\partial \tau_{r x}}, \quad \frac{\partial v}{\partial r}=\frac{\partial \mathrm{H}}{\partial \tau_{r \theta}}, \quad \frac{\partial w}{\partial r}=\frac{\partial \mathrm{H}}{\partial \sigma_{r}}, \\
& \frac{\partial \tau_{r x}}{\partial r}=-\frac{\partial \mathrm{H}}{\partial u}, \quad \frac{\partial \tau_{r \theta}}{\partial r}=-\frac{\partial \mathrm{H}}{\partial v}, \quad \frac{\partial \sigma_{r}}{\partial r}=-\frac{\partial \mathrm{H}}{\partial w},
\end{aligned}
$$

and one denotes

$$
q=\left(\begin{array}{lll}
u, & v, & w
\end{array}\right)^{T}, \quad p=\left(\begin{array}{lll}
\tau_{r x} & \tau_{r \theta} & \sigma_{r}
\end{array}\right)^{T}, \quad p_{l}=\left(\sigma_{x}, \sigma_{\theta}, \tau_{x \theta}\right)^{T}
$$

Equations (2.3) can also be written in a simplified form

$$
\frac{\partial q}{\partial r}=\frac{\partial \mathrm{H}}{\partial p}, \quad \frac{\partial p}{\partial r}=-\frac{\partial \mathrm{H}}{\partial q}
$$

This is the classical Hamilton canonical Eq.(3.6). Meanwhile, the following relations can be obtained

$$
\left.\begin{array}{l}
\iiint_{V}\left(\frac{\partial p}{\partial r}+\frac{\partial \mathrm{H}}{\partial q}\right) \delta q d V+\Gamma_{1}=0 \\
\iiint_{V}\left(\frac{\partial q}{\partial r}-\frac{\partial \mathrm{H}}{\partial p}\right) \delta p d V+\Gamma_{2}=0 \\
\iiint_{V}\left(D q-\frac{\partial \mathrm{H}}{\partial p_{1}}\right) \delta p_{1} d V+\Gamma_{3}=0
\end{array}\right\}
$$

where

$$
\Gamma_{l}=\left[\begin{array}{c}
\iint_{s_{\sigma}}\left(\bar{p}_{x}-p_{x}\right) \delta u d s=0 \\
\iint_{s_{\sigma}}\left(\bar{p}_{\theta}-p_{\theta}\right) \delta v d s=0 \\
\iint_{s_{\sigma}}\left(\bar{p}_{r}-p_{r}\right) \delta w d s=0
\end{array}\right], \quad \Gamma_{2}=\left[\begin{array}{c}
\iint_{s_{u}}\left[(\bar{w}-w) n_{x}+(\bar{u}-u) n_{r}\right] \delta \tau_{r x} d s=0 \\
\iint_{s_{u}}\left[(\bar{w}-w) n_{\theta}+(\bar{v}-v) n_{r}\right] \delta \tau_{r \theta} d s=0 \\
\iint_{s_{u}}(\bar{w}-w) n_{r} \delta \sigma_{r} d s=0
\end{array}\right],
$$




$$
\Gamma_{3}=\left[\begin{array}{c}
\iint_{s_{u}}(\bar{u}-u) n_{x} \delta \sigma_{x} d s=0 \\
\iint_{s_{u}}(\bar{v}-v) n_{\theta} \delta \sigma_{\theta} d s=0 \\
\iint_{s_{u}}\left[(\bar{v}-v) n_{x}+(\bar{u}-u) n_{\theta}\right] \delta \tau_{x \theta} d s=0
\end{array}\right], \quad D=\left[\begin{array}{ccc}
-\frac{\partial}{\partial x} & 0 & 0 \\
0 & -\frac{1}{r} \frac{\partial}{\partial \theta} & -\frac{1}{r} \\
-\frac{1}{r} \frac{\partial}{\partial \theta} & -\frac{\partial}{\partial x} & 0
\end{array}\right],
$$

Eqs (2.6) are weak formulations of mixed equations including boundary conditions.

\section{Solution of weak formulation}

An orthotropic thick closed cylindrical shell is investigated, and selecting $(\zeta=m \pi / l)$

$$
\begin{array}{ll}
u=\sum_{m} \sum_{n} u_{m n}(r) \cos (\zeta x) \cos (n \theta), & v=\sum_{m} \sum_{n} v_{m n}(r) \sin (\zeta x) \sin (n \theta), \\
w=\sum_{m} \sum_{n} w_{m n}(r) \sin (\zeta x) \cos (n \theta), & \tau_{r x}=\sum_{m} \sum_{n} \tau_{r x, m n}(r) \cos (\zeta x) \cos (n \theta), \\
\tau_{r \theta}=\sum_{m} \sum_{n} \tau_{r x, m n}(r) \sin (\zeta x) \sin (n \theta), & \sigma_{r}=\sum_{m} \sum_{n} \sigma_{r, m n}(r) \sin (\zeta x) \cos (n \theta), \\
T=\sum_{m} \sum_{n} T_{m n}(r) \sin \zeta x \cos (n \theta) . &
\end{array}
$$

Introducing Eqs (3.1) into Eq.(2.6), and letting

$$
\begin{aligned}
& \omega=\omega_{m n}, \quad C_{1}=-C_{13} / C_{33}, \quad C_{2}=C_{11}-C_{13}^{2} / C_{33}, \quad C_{3}=C_{12}-C_{13} C_{23} / C_{33}, \\
& C_{4}=C_{22}-C_{23}^{2} / C_{33}, \quad C_{5}=-C_{23} / C_{33}, \quad C_{7}=1 / C_{33}, \quad C_{8}=1 / C_{55}, \quad C_{9}=1 / C_{44}, \\
& C_{6}=C_{66}, \quad C_{a}=C_{3} \alpha_{x}+C_{4} \alpha_{\theta}, \quad C_{b}=C_{2} \alpha_{x}+C_{3} \alpha_{\theta}, \quad C_{d}=C_{1} \alpha_{x}+C_{5} \alpha_{\theta}-\alpha_{r},
\end{aligned}
$$

we obtain the mixed state Hamilton equation for the cylindrical shell for each combination of $m$ and $n$

$$
\frac{d}{d r} \boldsymbol{F}(r)=\boldsymbol{M}(r) \boldsymbol{F}(r)+\boldsymbol{B}(r)
$$

where

$$
\begin{aligned}
\boldsymbol{F}(r) & =\left[\begin{array}{llllll}
r u_{m n}(r) & r v_{m n}(r) & r w_{m n}(r) & \tau_{r x, m n}(r) & \tau_{r \theta, m n}(r) & \sigma_{r, m n}(r)
\end{array}\right]^{T}, \\
\boldsymbol{M}(r) & =\left[\begin{array}{cc}
\boldsymbol{A}^{T} & \boldsymbol{D} \\
\boldsymbol{E} & -\boldsymbol{A}
\end{array}\right],
\end{aligned}
$$




$$
\begin{aligned}
& \boldsymbol{A}^{T}=\left[\begin{array}{ccc}
\frac{1}{r} & 0 & -\zeta \\
0 & \frac{2}{r} & \frac{n}{r} \\
-C_{1} \zeta & \frac{C_{5} n}{r} & \frac{C_{5}+1}{r}
\end{array}\right], \quad \boldsymbol{D}=\boldsymbol{D}^{T}=\left[\begin{array}{ccc}
C_{8} r & 0 & 0 \\
0 & C_{9} r & 0 \\
0 & 0 & C_{7} r
\end{array}\right], \\
& \boldsymbol{E}=\boldsymbol{E}^{T}=\left[\begin{array}{ccc}
\frac{C_{2}}{r} \zeta^{2}+\frac{C_{6}}{r^{3}} n^{2}-\frac{p_{x} \zeta^{2}}{r} & -\frac{C_{3}+C_{6}}{r^{2}} \zeta n & -\frac{C_{3}}{r^{2}} \zeta \\
-\frac{C_{3}+C_{6}}{r^{2}} \zeta n & \frac{C_{6}}{r} \zeta^{2}+\frac{C_{4}}{r^{3}} n^{2}-\frac{p_{x} \zeta^{2}}{r} & \frac{C_{4}}{r^{3}} n \\
-\frac{C_{3}}{r^{2}} \zeta & \frac{C_{4}}{r^{3}} n & \frac{C_{4}}{r^{3}}-\frac{p_{x} \zeta^{2}}{r}
\end{array}\right], \\
& \boldsymbol{B}(r)=\left[\begin{array}{llllll}
0 & 0 & -C_{d} r T_{m n}(r) & \zeta C_{b} T_{m n}(r) & -\frac{n C_{a} T_{m n}(r)}{r} & -\frac{C_{a} T_{m n}(r)}{r}
\end{array}\right]^{T} .
\end{aligned}
$$

In order to solve Eq.(3.3), the thick shell should be divided into some thin plies. If we find, from calculation, that the needful effective digits hardly change, it can be said that the results obtained with certain thin plies are exact within the prescribed accuracy limits. For the first ply, the solution of Eq.(3.4) is

$$
\boldsymbol{F}(r)=\boldsymbol{G}(r-a) \boldsymbol{F}(a)+\boldsymbol{C}(r-a),
$$

in which

$$
\boldsymbol{G}(r-a)=\exp [\boldsymbol{M} \cdot(r-a)], \quad \boldsymbol{C}(r-a)=\int_{r} \exp [\boldsymbol{M} \cdot(r-\tau)] \boldsymbol{B}(\tau) d \tau
$$

Equation (3.5) is a Hamiltonian matrix. The present result is exactly analogous to the Hamiltonian mechanics for a dynamic system. Because $\boldsymbol{M}$ is a Hamiltonian matrix, $\boldsymbol{G}(r-a)$ can be calculated by means of symplectic algorithm (Ding, 2000; 2009).

In order to calculate a multiple shell, we can apply the transfer matrix method, for the first ply we have ( $h_{l}$-thickness of the first ply)

$$
\boldsymbol{F}\left(r_{l}\right)=\boldsymbol{G}\left(-h_{l}\right) \boldsymbol{F}(a)+\boldsymbol{C}\left(-h_{l}\right) .
$$

By virtue of the continuity conditions for displacements and stresses between the first and second ply, there must be

$$
\boldsymbol{F}\left(r_{2}\right)=\boldsymbol{G}\left(-h_{2}\right) \boldsymbol{G}\left(-h_{1}\right) \boldsymbol{F}(a)+\boldsymbol{G}\left(-h_{2}\right) \boldsymbol{C}\left(-h_{1}\right)+\boldsymbol{C}\left(-h_{2}\right) .
$$

Similarly, the mechanical quantities of the interior surface and outer surfaces for the entire laminated shell can be linked together to be of the form ( $k$ - the number of layers) 


$$
\boldsymbol{F}(b)=\Pi \boldsymbol{F}(a)+\bar{\Pi}
$$

in which

$$
\begin{aligned}
& \boldsymbol{\Pi}=\boldsymbol{G}\left(-h_{k}\right) \boldsymbol{G}\left(-h_{k-1}\right) \boldsymbol{G}\left(-h_{k-2}\right) \ldots \ldots \boldsymbol{G}\left(-h_{2}\right) \boldsymbol{G}\left(-h_{1}\right), \\
& \overline{\boldsymbol{\Pi}}=\boldsymbol{G}\left(-h_{k}\right) \sum_{j=l}^{k-1}\left[\prod_{i=k-1}^{j+1} \boldsymbol{G}\left(-h_{i}\right) \boldsymbol{C}\left(-h_{j}\right)\right]+\boldsymbol{C}\left(-h_{k}\right) .
\end{aligned}
$$

Actually, Eq.(3.8) is a matrix equation for six displacements of the outer and interior surfaces of a shell. $\Pi$ is a $(6 \times 6)$ constant matrix. Usually, the loads acting on the interior and outer surfaces of a shell are given a priori. Actually, Eq.(3.8) is a matrix equation for four displacements of the outer and interior surfaces of a shell. When a normal pressure $q$ acts on the interior surface of the shell, expanding the load in the same series as the $\sigma$-series in Eq.(3.1), one has

$$
\sigma_{r, m n}(a)=\tau_{r x, m n}(a)=\tau_{r \theta, m n}(a)=\sigma_{r, m n}(b)=\tau_{r x, m n}(b)=\tau_{r \theta, m n}(b)=0 .
$$

Selecting the third, fourth and fifth rows of matrix Eq.(3.8) gives

$$
\left[\begin{array}{ccc}
\Pi_{31} & \Pi_{32} & \Pi_{36} \\
\Pi_{41} & \Pi_{42} & \Pi_{46} \\
\Pi_{51} & \Pi_{52} & \Pi_{56}
\end{array}\right]\left\{\begin{array}{c}
u_{m n}(a) \\
v_{m n}(a) \\
w_{m n}(a)
\end{array}\right\}=\left\{\begin{array}{c}
\sigma_{m}(b) \\
0 \\
0
\end{array}\right\} .
$$

$u_{m n}(a), \quad v_{m n}(a)$ and $w_{m n}(a)$ can be determined by Eq.(3.11). After finding these quantities, Eq.(3.6) is employed, other unknown coefficients can be solved. Reasoning by analogy, the quantities in the first layer are solved. In the same way, the calculations for the mechanical quantities of the entire laminated shell can easily be programmed.

In order to solve the buckling problem, let the right hand side of Eq.(3.11) be zero, the nontrivial solution of Eq.(3.11) gives

$$
\left[\begin{array}{lll}
\Pi_{41} & \Pi_{42} & \Pi_{43} \\
\Pi_{51} & \Pi_{52} & \Pi_{53} \\
\Pi_{61} & \Pi_{62} & \Pi_{63}
\end{array}\right]=\left\{\begin{array}{l}
0 \\
0 \\
0
\end{array}\right\} .
$$

In fact, Eq.(3.12) is the exact critical stress equation of the laminated shell in the sense of satisfying a prescribed precision. An infinite number of critical stresses can be obtained from Eq.(3.12). Of course, the minimum critical stress is the most useful.

\section{Numerical example}

Example. Consider the thermoelastic buckling analysis of a 3-plied closed cylindrical shell to the temperature variation $T=T_{1} \sin \frac{\pi x}{l} \cos \theta$. (Fig.1). The materials for the first and third layers are identical. Each layer has the same elastic constants 


$$
\begin{array}{lll}
C_{12} / C_{11}=0.246269, & C_{13} / C_{11}=0.0831715, & C_{22} / C_{11}=0.543103, \\
C_{23} / C_{11}=0.115017, & C_{33} / C_{11}=0.530172, & C_{44} / C_{11}=0.266810, \\
C_{55} / C_{11}=0.159914, & C_{66} / C_{11}=0.262931, & C_{11}^{(1)} / C_{11}^{(2)}=5
\end{array}
$$

where $C_{11}^{(1)}$ and $C_{11}^{(2)}$ denote $C_{11}$ of the materials corresponding to the first and second layer, respectively. The laminated shell has the following geometry parameters

$$
h_{l}=h_{3}=0.1 h, \quad h_{2}=0.8 h, \quad l=s=2 \pi R_{\circ}
$$

where $l=$ the length of the shell, $s=$ the arc length of middle surface and $R=$ the radius of middle surface. When $m=n=1$, the first three critical stresses for the single-ply shell $\left(C_{11}^{(1)}=C_{11}^{(2)}=C_{11}\right)$ and the three-plied shell are indicated in Tab.1. The results for the three-dimensional finite element method (FEM) using SAP5 are also shown in Tab.1. Because of the symmetry, 24 three-dimensional isoparametric elements (for $1 / 4$ shell) with 20 nodes are employed in the calculation. It can be seen that the first-order critical stresses calculated by SAP5 are accurate enough. Where $\lambda=(\pi h / l) \sqrt{\rho_{x c r} / C_{11}}, \quad\left(\rho_{x c r} / C_{11}\right)_{i}=\operatorname{constan} t$, $i=1,2,3, \rho_{x c r}=$ critical stress.

Table 1. Critical stress parameters $\lambda$ for a single-ply shell and a three-plied laminated shell with different ratios $h / R_{0}$.

\begin{tabular}{|c|c|c|c|c|c|c|c|}
\hline \multirow{2}{*}{$h / R_{0}$} & SAP5 & \multicolumn{3}{|c|}{ present single-ply } & \multicolumn{3}{c|}{ present three-plied } \\
\cline { 2 - 8 } & $\lambda_{1}$ & $\lambda_{1}$ & $\lambda_{2}$ & $\lambda_{3}$ & $\lambda_{1}$ & $\lambda_{2}$ & $\lambda_{3}$ \\
\hline 0.1 & 0.0126 & 0.0124 & 0.06482 & 0.1092 & 0.0123 & 0.0649 & 0.1078 \\
\hline 0.2 & 0.0249 & 0.0247 & 0.1311 & 0.2160 & 0.0248 & 0.1311 & 0.2139 \\
\hline 0.8 & - & 0.1063 & 0.5281 & 0.7888 & 0.1097 & 0.5189 & 0.7341 \\
\hline 1.0 & - & 0.1372 & 0.6849 & 0.9396 & 0.1415 & 0.6311 & 0.8551 \\
\hline
\end{tabular}

\section{Conclusion}

Weak formulations of mixed state equations of closed laminated cylindrical shells are presented in the Hamilton System. The Hamilton canonical equation of a closed cylindrical shell is established. A threedimensional thermoelastic solution is expressed for the buckling analysis of the thick closed laminated shell of an arbitrary thickness. Numerical results are obtained and compared with those of FEM calculated using SAP5. The present study satisfies the continuity conditions of stresses and displacements at the interfaces. Solutions and method such as this have value for designing laminated composite structures in naval, aerospace and other engineering applications.

\section{Acknowledgments}

This project 11472005 supported by National Natural Science Foundation of China and Anhui Provincial Science and Technology Research Project Funding through grant No.1501041133. 


\section{Nomenclature}

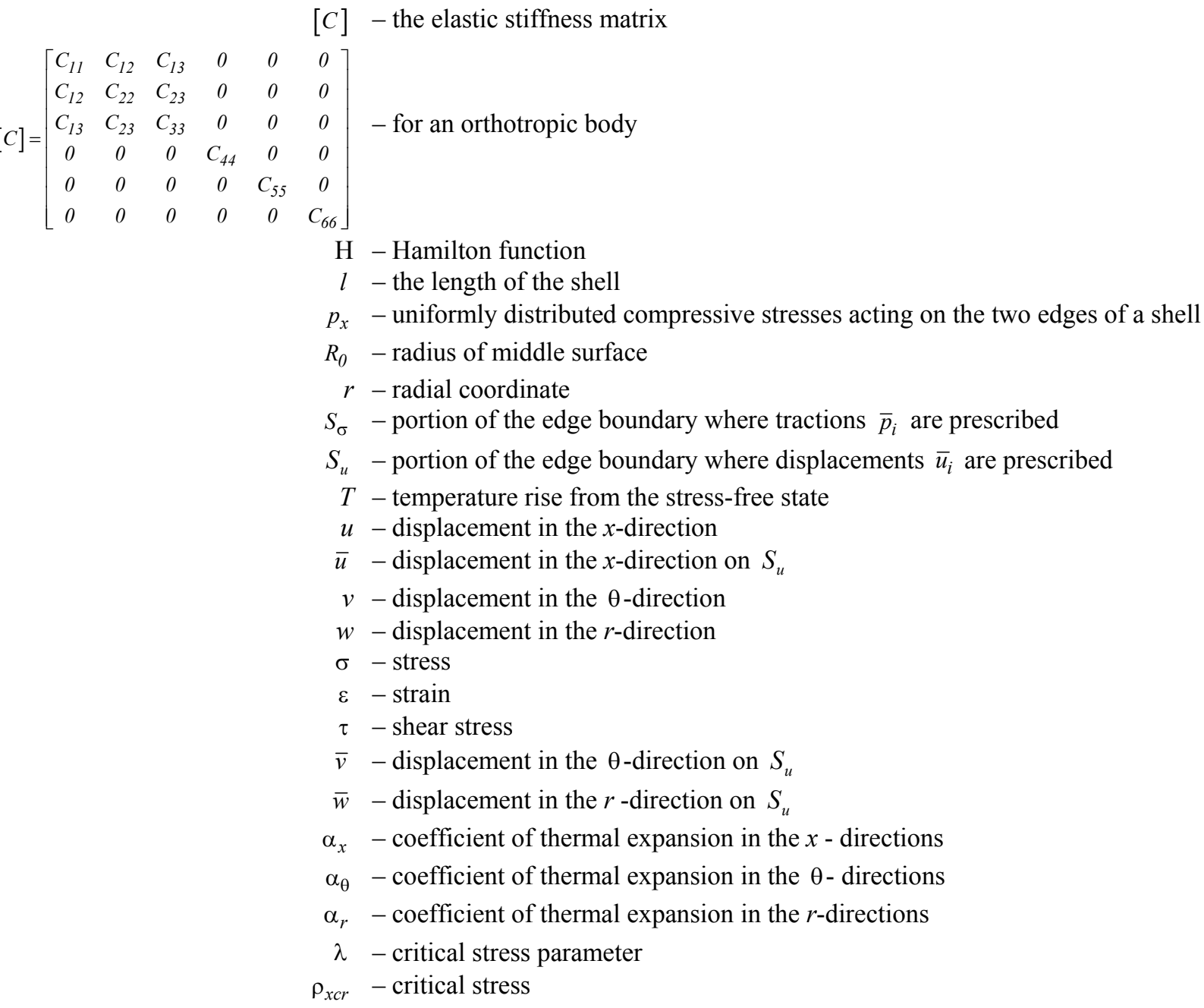

\section{References}

Brischetto S. (2009): Effect of the through-the-thickness temperature distribution on the response of layered and composite shells. - International Journal of Applied Mechanics, vol.1, No.4, pp.581-605.

Burgueño Rigoberto, Hu Nan, Heeringa Annelise and Lajnef Nizar (2014): Tailoring the elastic postbuckling response of thin-walled cylindrical composite shells under axial compression. - Thin-Walled Structures, vol.84, pp.14-25.

Chen W.C. and Liu W.H. (1993): Thermal buckling of antisymmetric angle-ply laminated plates an analytical levy-type solution. - Journal of Thermal Stresses, vol.16, pp.401-419.

Civalek and Omer (2013): Vibration analysis of laminated composite conical shells by the method of discrete singular convolution based on the shear deformation theory. - Composites Part B: Engineering, vol.45, No.1, pp.1001-1009.

Ding Kewei (2000): Symplectic algorithm method for Hamiltonian matrix eigenvalue and eigenvector problem. Journal of Hefei University of Technology, vol.23, No.3, pp.336-340 (in Chinese). 
Ding Kewei (2005): Weak formulation study of thick laminated cylindrical shells. - Shell Structures, Theory and Applications Proceedings of the 8th International Conference on Shell Structures (SSTA 2005), Jurata, Gdansk, Poland, pp. Taylor \& Francis, London, pp.97-100.

Ding Kewei (2008): Weak formulation study for thermoelastic analysis of thick open laminated shell. - Mechanics of Advanced Materials and Structures, vol.15, pp.33-39.

Ding Kewei (2008): Thermal stresses of weak formulation study for thick laminated shell. - Journal of Thermal Stresses, vol.31, No.4, pp.389-400.

Ding Kewei (2009): Establishment of weak formulation for the dual system of Hamilton equations. - Journal of Anhui University of Science and Technology, vol.29, No.3, pp.26-30 (in Chinese).

Ding Kewei and Tang Limin (1997): Exact analysis for axisymmetric vibration and buckling of the thick laminated closed cylindrical shells in a Hamilton system. - Journal of Sound and Vibration, vol.206, No.3, pp.435-441.

Ding Kewei and Tang Limin (1998): Exact thermoelastic solution for axisymmetric problem of thick closed laminated shells. - Journal of Thermal Stresses, vol.21, No.7, pp.751-761.

Ding Kewei and Tang Limin (1999): Three-dimensional free vibration of thick laminated cylindrical shells with clamped edges. - Journal of Sound and Vibration, vol.220, No.1, pp.171-177.

Ding Kewei and Tang Limin (1999): Exact solution for axisymmetric thick laminated shells. - Composite Structures, vol.46, No.2, pp.125-129.

Hashemian A.H. and Jam J.E. (2010): Nonlinear free dynamic response of laminated compressible cylindrical shell panels. - Mechanics of Composite Materials, vol.46, No.1, pp.15-28.

Hawkes T.D. and Soldatos K.P. (1992): Three-dimensional axisymmetric vibrations of orthotropic and cross-ply laminated hollow cylinders. - AIAA J., vol.30, pp.1089-1098.

Hedayati H., Hedayati M., Aragh B. Sobhani and Farahani E. Borzabadi (2014): Two-dimensional differential quadrature solution for vibration characteristics of two-dimensional functionally graded metal/ceramic open cylindrical shells. - Mechanics of Advanced Materials and Structures, vol.21, No.4, pp.305-320.

Huang N.N. and Tauchert T.R. (1992): Thermal Stresses in Doubly-Curved Cross-Ply Laminates. - International Journal of Solids and Structures, vol.29, pp.991-1000.

Kardomateas G.A. (1993): Buckling of thick orthotropic cylindrical shells under external pressure. - ASME J. Appl. Mech., vol.60, pp.195-202.

Khdeir A.A. (1996): Thermoelastic analysis of cross-ply laminated circular cylindrical shells. - International Journal of Solids and Structures, vol.33, No.27, pp.4007-4017.

Khdeir A.A. (2012): Thermoelastic response of cross-ply laminated shells based on a rigorous shell theory. - Journal of Thermal Stresses, vol.35, No.11, pp.1000-1017.

Maghami S.A. and Tahani M. (2015): Thermal bending analysis of doubly curved composite laminated shell panels with general boundary conditions and laminations. - Journal of Thermal Stresses, vol.38, No.2, pp.250-270.

Noor A.K. and Burton W.S. (1996): Computational models for sandwich panels and shells. - Applied Mechanics and Review, vol.49 No.3, pp.155-199.

Reddy J.N. (1983): Dynamics analysis of layered anisotropic composite material plates. - Int. J. Num. Mech. Engng., vol.192, pp.237-255.

Reddy J.N. (1984): Exact solution of moderately thick laminated shells. - Journal of Engineering Mechanics Division, vol.111, pp.794-809.

Soldatos K.P. and Hadjigeorgiou V.P. (1990): Three-dimensional solution of the free vibration problem of homogeneous isotropic cylindrical shells and panels. - J. Sound Vibration, vol.137, pp.369-384.

Soldatos K.P. and Ye J.Q. (1994): Three-Dimensional Static, Dynamic, Thermoelastic and Buckling Analysis of Homogeneous and Laminated Composite Cylinders. - Composite Structures, vol.29, pp.131-143. 
Srinivas S. and Rao A.K. (1970): Bending, vibration and buckling of simply supported thick orthotropic rectangular plates and laminates. - International Journal of Solids and Structures, vol.6, pp.1463-1481.

Topal Umut and Uzman Umit (2011): Frequency optimization of laminated skewed open cylindrical shells. - Science and Engineering of Composite Materials, vol.18, No.3, pp.139-144.

Yao Guo and Li Feng-Ming (2014): The stability analysis and active control of a composite laminated open cylindrical shell in subsonic airflow. - Journal of Intelligent Material Systems and Structures, vol.25, No.3, pp.259-270.

Received: June 5, 2015

Revised: June 9, 2015 